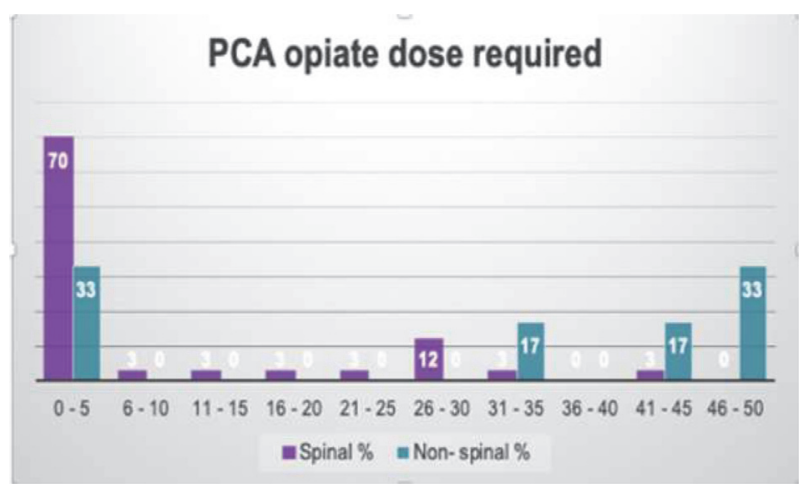

Abstract 212 Figure 2

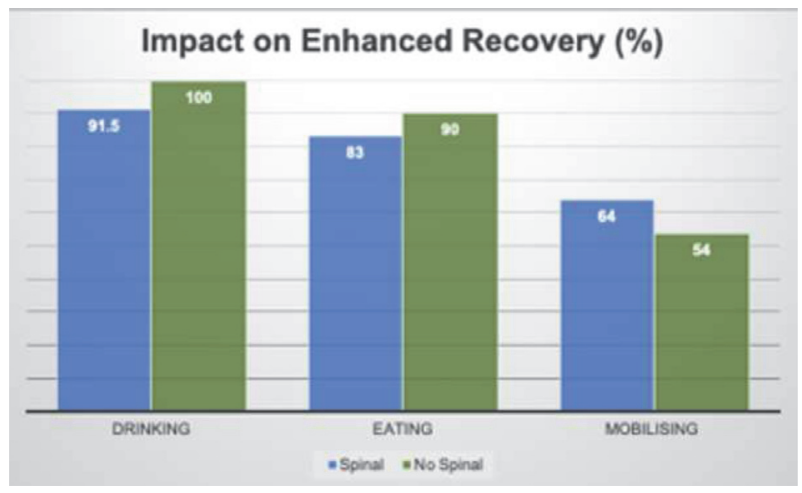

Abstract 212 Figure 3

Conclusions We demonstrated that intrathecal diamorphine reduced post-operative opiate requirements and facilitated earlier mobilisation.

We concluded that the use of intrathecal diamorphine in combination with multimodal analgesia in colorectal surgery is safe and is comparable, if not marginally superior, to PCA in our institution.

We have also shown that despite a wide range of intrathecal diamorphine dosage $(0.5-1 \mathrm{mg})$, there were no post-operative complications which is in keeping with anecdotal experience. Therefore, we feel that intrathecal diamorphine for perioperative analgesia for resectional bowel surgery is a safe and viable technique.

\section{PAIN MANAGEMENT AFTER LAMINECTOMY: A SYSTEMATIC REVIEW AND PROCEDURE-SPECIFIC POST-OPERATIVE PAIN MANAGEMENT (PROSPECT) RECOMMENDATIONS}

${ }^{1} \mathrm{~L}$ Peene ${ }^{*},{ }^{2} \mathrm{P}$ Le Cacheux, ${ }^{3,4} \mathrm{AR}$ Sauter, ${ }^{5} \mathrm{GP}$ Joshi, ${ }^{2} \mathrm{H}$ Beloeil. ${ }^{1}$ University Hospitals Leuven, Leuven, Belgium; ${ }^{2} \mathrm{CHU}$ Rennes, Rennes, France; ${ }^{3}$ Oslo University Hospital, Oslo, Norway; ${ }^{4}$ Inselspital, Bern University Hospital, Bern, Switzerland; ${ }^{5}$ University of Texas Southwestern Medical Center, Dallas, USA

\subsection{6/rapm-2021-ESRA.213}

Background and Aims With lumbar laminectomy increasingly being performed on an outpatient basis, optimal pain management is critical to avoid post-operative delay in discharge and readmission. [1, 4] The aim of this review was to evaluate the available literature and develop recommendations for optimal pain management after one- or two-level lumbar laminectomy.
Methods A systematic review utilizing the PROcedure-SPECific Post-operative Pain ManagemenT (PROSPECT) methodology was undertaken [5]. Randomised controlled trials (RCTs) published in the English language from 1 January 2008 until 31 March 2020 - assessing post-operative pain using analgesic, anaesthetic and surgical interventions - were identified from MEDLINE, EMBASE and Cochrane Databases.

Results Out of 65 eligible studies identified, 39 RCTs met the inclusion criteria. The analgesic regimen for lumbar laminectomy should include paracetamol and a non-steroidal antiinflammatory drug (NSAID) or cyclooxygenase (COX)-2 selective inhibitor administered preoperatively or intraoperatively and continued post-operatively, with post-operative opioids for rescue analgesia. In addition, surgical wound instillation or infiltration with local anaesthetics prior to wound closure is recommended (table 1). Some interventions - gabapentinoids and intrathecal opioid administration - although effective, carry significant risks and consequently were omitted from the recommendations. Other interventions were also not recommended because there was insufficient, inconsistent or lack of evidence (table 2).

Abstract 213 Table 1 Overall recommendations for perioperative pain management in patients undergoing lumbar laminectomy

Preoperative and intraoperative recommendations
Oral or IV paracetamol (Grade D)
Oral or IV NSAIDs/COX-2-specific inhibitors (Grade A)
Surgical wound instillation or infiltration with local anaesth
(Grade A)
Post-operative mcommendations
Oral or IV paracetamol (Grade D)
Oral or IV NSAIDs/COX-2-specific inhibitors (Grade A)
Opioids as rescue medication (Grade D)

COX-2, cyclooxygenase-2; IV, intravenous; NSAIDs, non-steroidal anti-inflammatory drugs

Table 2 Analgesic interventions that are not recommended for pain management in patients undergoing lunbar laminectomy

\begin{tabular}{|c|c|}
\hline Intervention & Reason for not recommending \\
\hline Dexamethasone & $\begin{array}{l}\text { Limited procedure-specific } \\
\text { evidence }\end{array}$ \\
\hline Oral gabapentin/pregabalin & Significant risk for adverse effects \\
\hline Intrathecal opioids & Sig̨nificant risk for adverse effects \\
\hline Epidural analgesia & $\begin{array}{l}\text { Limited procedure-specific } \\
\text { evidence and risk for adverse } \\
\text { effects }\end{array}$ \\
\hline Paravertebral block & $\begin{array}{l}\text { Limited procedure-specific } \\
\text { evidence }\end{array}$ \\
\hline Surgical perineural infiltration & $\begin{array}{l}\text { Limited procedure-specific } \\
\text { evidence }\end{array}$ \\
\hline Surgical wound local infiltration & $\begin{array}{l}\text { Linited procedure-specific } \\
\text { evidence }\end{array}$ \\
\hline Corticosteroids & $\begin{array}{l}\text { Limited procedure-specific } \\
\text { evidence }\end{array}$ \\
\hline Intravenous magnesium & $\begin{array}{l}\text { Lack of procedure-specific } \\
\text { evidence }\end{array}$ \\
\hline Transdermal fentany! & $\begin{array}{l}\text { Limited procedure-specific } \\
\text { evidence and risk for adverse } \\
\text { effects }\end{array}$ \\
\hline
\end{tabular}


Abstract 213 Table 2 Analgesic interventions that are not recommended for pain management in patients undergoing lumbar laminectomy

\section{Preoperative and intraoperative recommendations \\ Oral of IV paracetamol (Grade D) \\ Oral or IV NSAIDv/COX-2-specific inhibitors (Grade A) \\ Surgical wound instillation or infiltration with local anaesthetic (Grade A) \\ Post-operative mcommendations \\ Oral or IV paracetamol (Grade D) \\ Oral of IV NSAIDs/COX-2-specific inhibitors (Grade A) \\ Opioids as rescue medication (Grade D)}

COX-2, cyclooxygenase-2; IV, intravenous; NSAIDs, non-steroidal anti-inflammatory drugs

Table 2 Analgesic interventions that are not recommended for pain management in patients undergoing lunbar laminectomy

\begin{tabular}{|c|c|}
\hline Intervention & Reason for not recommending \\
\hline Dexamethasone & $\begin{array}{l}\text { Limited procedure-specific } \\
\text { evidence }\end{array}$ \\
\hline Oral gabapentin/pregabalin & Significant risk for adverse effects \\
\hline Intrathecal opioids & Significant risk for adverse effects \\
\hline Epidural analgesia & $\begin{array}{l}\text { Limited procedure-specific } \\
\text { evidence and risk for adverse } \\
\text { effects }\end{array}$ \\
\hline Paravertebral block & $\begin{array}{l}\text { Limited procedure-specific } \\
\text { evidence }\end{array}$ \\
\hline Surgical perineural infiltration & $\begin{array}{l}\text { Limited procedure-specific } \\
\text { evidence }\end{array}$ \\
\hline Surgical wound local infiltration & $\begin{array}{l}\text { Linited procedure-specific } \\
\text { evidence }\end{array}$ \\
\hline Corticosteroids & $\begin{array}{l}\text { Linited procedure-specific } \\
\text { evidence }\end{array}$ \\
\hline Intravenous magnesium & $\begin{array}{l}\text { Lack of procedure-specific } \\
\text { evidence }\end{array}$ \\
\hline Transdermal fentanyl & $\begin{array}{l}\text { Limited procedure-specific } \\
\text { evidence and risk for adverse } \\
\text { effects }\end{array}$ \\
\hline
\end{tabular}

Conclusions Perioperative pain management for lumbar laminectomy should include paracetamol and NSAID- or COX-2specific inhibitor, continued into the post-operative period, as well as intraoperative surgical wound instillation or infiltration. Opioids should be used as rescue medication post-operatively. Future studies are necessary to evaluate the efficacy of our recommendations.

\section{VIRTUAL REALITY HYPNOSIS ON COLD PAIN PERCEPTION IN HEALTHY VOLUNTEERS}

${ }^{1} \mathrm{C}$ Terzulli* ${ }^{2} \mathrm{C}$ Chauvin, ${ }^{3} \mathrm{C}$ Champagnol Di-Liberti, ${ }^{3} \mathrm{~S}$ Faisan, ${ }^{4} \mathrm{~A}$ Dufour, ${ }^{1} \mathrm{M}$ Melchior, ${ }^{3} \mathrm{~L}$ Goffin, ${ }^{5} \mathrm{D}$ Graff, ${ }^{3} \mathrm{E}$ Laroche, ${ }^{6} \mathrm{E}$ Salvat, ${ }^{1} \mathrm{P}$ Poisbeau. ${ }^{1}$ Institut des Neurosciences Cellulaires et Intégratives, Strasbourg, France; ${ }^{2}$ Hôpital de Hautepierre, Strabourg, France; ${ }^{3}$ ICUBE, Strasbourg, France; ${ }^{4}$ Centre d'Investigation Neurocognitives et Neurophysiologiques, Strasbourg, France; ${ }^{5}$ Clinique Rhéna, Strasbourg, France; ${ }^{6}$ CETD; Hôpital de Hautepierre, Strabourg, France
Background and Aims Over 200 millions of surgeries are performed each year, these may lead to postoperative pain mainly managed with opioid prescription [1]. It is recommended to decrease the use of opioids by adding at least one non-pharmacological pain management modality in the treatment [2]. Virtual Reality and hypnosis are examples of such.

The aim of this study was to measure the pain intensity and unpleasantness perception at rest and during virtual reality hypnosis (VRH) in healthy volunteers.

Abstract 214 Table 1 Demographic data of the 41 healthy volunteers included

\begin{tabular}{|c|c|c|c|c|c|c|c|}
\hline & Shate, s) & (new) & $\begin{array}{l}\text { Iducation } \\
\text { treent) }\end{array}$ & ${ }_{\mathrm{vin}}$ & $\begin{array}{l}\text { swe then } \\
\text { Viag }\end{array}$ & 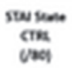 & 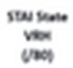 \\
\hline mat & 53 & 41,28 & 4.80 & 5.97 & 16.55 & 28.17 & 22,43 \\
\hline so & & 200 & 200 & 102 & 869 & 5.58 & 14 \\
\hline
\end{tabular}
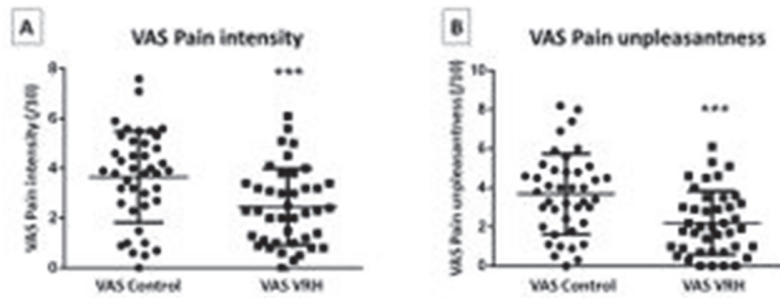

Abstract 214 Figure 2

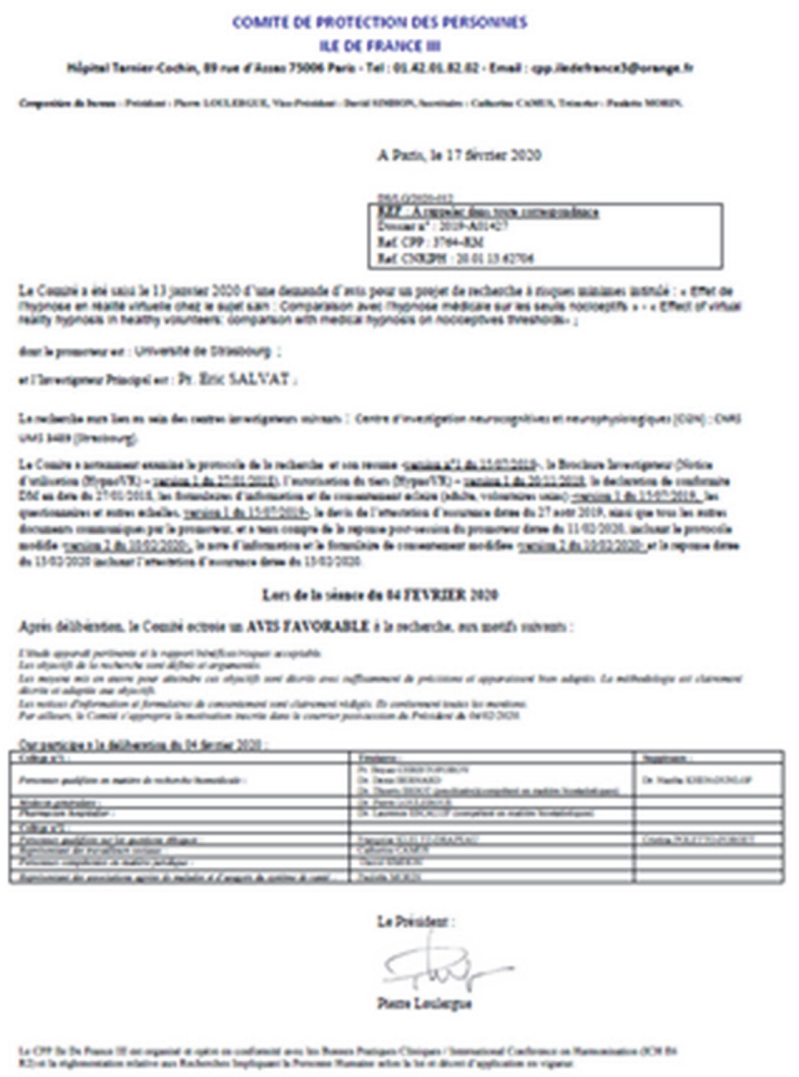

Abstract 214 Figure 1 